\title{
Effects of environmental endocrine disruptors, including insecticides used for malaria vector control on reproductive parameters of male rats
}

\author{
Sean M. Patrick ${ }^{1,2}$, Maria S. Bornman ${ }^{1,2}$, Annie M. Joubert ${ }^{3}$, Neville Pitts ${ }^{4}$, Vinny \\ Naidoo $^{2,5}$, Christiaan de Jager ${ }^{1,2}$ \\ ${ }^{1}$ School of Health Systems and Public Health, University of Pretoria \\ 2 University of Pretoria Center for Sustainable Malaria Control (UP CSMC) \\ 3 Department of Physiology, University of Pretoria \\ ${ }^{4}$ School of Physiology, University of the Witwatersrand \\ ${ }^{5}$ Biomedical Research Centre, Faculty of Veterinary Science, University of Pretoria
}

\section{Abstract}

The male reproductive system is sensitive to endocrine disrupting chemicals (EDCs) during critical developmental windows. Male Sprague-Dawley rats were exposed in utero-, during lactation- and directly to 1,1,1-trichloro-2,2-bis(p-chlorophenyl)ethane (DDT), 1,1,-dichloro2,2-bis(p-chlorophenyl)ethylene (DDE) and a mixture of DDT, deltamethrin (DM), pnonylphenol $(p-N P)$ and phytoestrogens, at concentrations found in a malaria-area. After dosing for 104 days, histological assessments and reproductive-endpoints were assessed. The anogenital distance (AGD) $(P=0.005)$ was shorter in the mixture-exposed group, while the prostate mass $(P=0.018)$ was higher in the DDT-exposed group. A higher testicular mass and abnormal histology was observed in the DDT- $(P=0.019)$, DDE- $(P=0.047)$ and mixture-exposed $(P<0.005)$ groups. This study shows that in utero-, lactational- and direct exposure to EDCs present in a malaria-area negatively affects male reproductive parameters in rats. These findings raise concerns to EDC-exposures to mothers living in malaria-areas and the reproductive health of their male offspring. 
Key Words: DDT, DDE, deltamethrin, p-Nonylphenol, phytoestrogens, endocrine disrupting chemicals, South Africa

\section{Abbreviations}

Endocrine disrupting chemicals (EDCs)

1,1,1-trichloro-2,2-bis(p-chlorophenyl)ethane (DDT)

1,1,-dichloro-2,2-bis(p-chlorophenyl)ethylene (DDE)

deltamethrin (DM)

$p$-nonylphenol ( $p$-NP)

Anogenital distance (AGD)

\section{Introduction}

Endocrine disrupting chemicals (EDCs) are ubiquitous in the environment and interfere with physiological processes through interactions with nuclear hormone receptors [1]. Hormonal regulation in the reproductive system can be disrupted following exposure to EDCs [2]. Disrupting hormone dependent processes during the sensitive critical developmental windows of gestation may affect development and maturation later in life [3]. The male reproductive system, particularly the testes, is sensitive to hormonal disruption as it is the site of androgen synthesis. Insufficient androgens in laboratory animals caused by exposure to EDCs may induce reproductive abnormalities [4], including feminization of males, altered sex ratios and impaired spermatogenesis [5]. However, the association between exposure to environmentally relevant concentrations of EDCs and impaired reproductive health remains unclear[1].

In South Africa, malaria is a public health threat and various programs are in place to prevent malaria transmission. The Vhembe district of South Africa is characterized by malaria and extensive agricultural activity. Relevant EDCs found malaria areas [6-11] have 
been identified as: 1,1,1-trichloro-2,2-bis(p-chlorophenyl)ethane (DDT), 1,1,-dichloro-2,2bis(p-chlorophenyl) ethylene (DDE), deltamethrin (DM), p-nonylphenol ( $p$-NP) and phytoestrogens (coumestrol, genistein and zearalenone - which have been linked to normal dietary intake). The organochlorine insecticide, DDT, has effectively been used to control mosquitoes in malaria endemic regions, including South Africa [12]. DDT is an EDC with estrogenic properties [2]. The main metabolite of DDT, $p, p^{\prime}-\mathrm{DDE}$, has anti-androgenic properties - inhibiting the action of natural androgens and binding to receptors [13]. DM is a synthetic pyrethroid, with estrogenic properties and is currently used for malaria indoor residual spraying (IRS) in western type houses and to treat insecticide-treated nets (ITNs) used in malaria vector control programs [14]. Other estrogen agonists are found in environmental ground water, such as $p$-NP, an anti-oxidant with multiple sources. It is used in the preparation of lubricating oil additives, as a plasticizer in the food packaging industry and is used in the processing of agricultural chemicals [15]. High levels of $p$-NP cause impaired reproductive development and decreased fertility potential in rats [16]. Phytoestrogens, genistein and coumestrol, are found in maize and beans which are important food crops in many malaria areas in South Africa [17]. Zearalenone is a nonsteroidal mycotoxin with estrogenic activity commonly found in maize, wheat, barley and rye. Tropical areas, such as the Vhembe district in South Africa, provide the ideal conditions for zearalenone to invade crops [17].

The "something from nothing" principle proposes that exposure to a single chemical may have no observed effects, but exposure to several of those chemicals in a mixture, due to synergistic or additive effects, may be significant [18]. During development, exposure to chemicals during the critical androgen-sensitive windows may result in altered embryonic patterning and/or a negative impact on the reproductive system development [2]. Assessing the effects of individual chemicals or mixtures on reproductive development requires laboratory studies of controlled exposures in a regulated environment.

This study investigates the effects of life time exposure (in utero-, lactational- and direct) to a mixture of environmentally relevant EDC concentrations. The response of male 
reproductive parameters, testicular histology, and associated hormonal changes in SpragueDawley rats to EDCs found in the Vhembe district of South Africa is presented.

\section{Materials and Methods}

\subsection{Study design}

Ethical clearance was obtained by the Animal Use and Care Committee of the University of Pretoria prior to the commencement of the project (Project number: H010/11) and in accordance with the South African code for the use and care of animals in research (SANS 10386) [19]. The original Organization for Economic Cooperation and Development (OECD) one generation reproductive toxicology 415 protocol [20] was modified to include a longer prenatal exposure period and additional male specific endocrine sensitive endpoints (Fig 1).

\subsection{Animals and housing conditions}

The study was performed at the University of Pretoria Biomedical Research Centre (UPBRC) using twenty-four pregnant female Sprague-Dawley rats. Animals were housed according to standard procedures with 12-h-day/night cycles, constant temperature (21 \pm 2 $\left.{ }^{\circ} \mathrm{C}\right)$ and humidity $(45 \% \pm 10 \%)$ in standard poly-carbonate Eurostandard type III cages, as per standard operating procedures. The animals were maintained on a diet of rodent pellets (Epol rodent cubes, Pretoria, South Africa) and high-pure water. Animals had free access to both food and water.

\subsection{Experimental design}

Twenty-four pregnant females (six pregnant females per group) were assigned into 4 groups and allowed to acclimatize for 3 days, prior to dosing. 


\section{Indirect Gestational and Lactational Exposure}

P1 females mated (Total $n=24)$

v

7 Day pregnant females

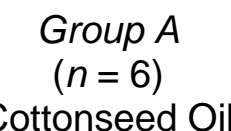

Group B
$(n=6)$
DDT $(35 \mathrm{mg} / \mathrm{kg})$

$\downarrow$

Cottonseed Oil

Group A

$(n=24)$

Cottonseed Oil

$$
\begin{aligned}
& \text { DDE (35 mg/kg) } \\
& \downarrow
\end{aligned}
$$

\section{Direct Exposure}

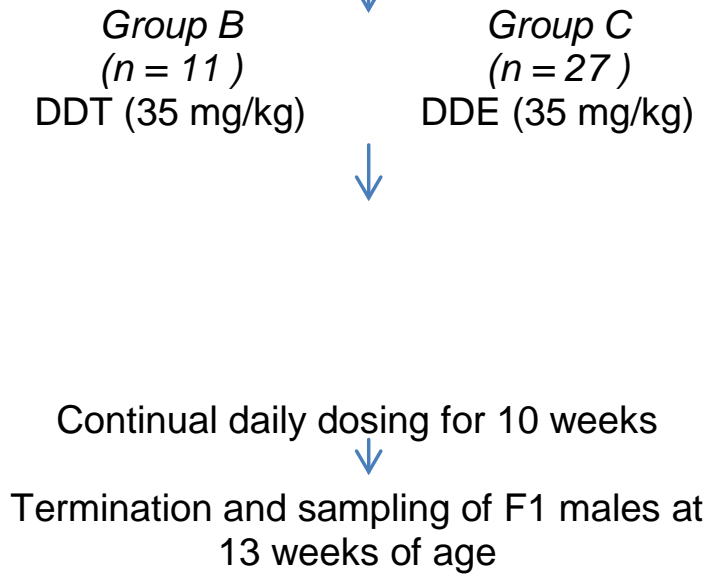

Continual daily dosing for 10 weeks

Termination and sampling of F1 males at 13 weeks of age$$
\text { Group C }
$$$$
(n=6)
$$$$
\text { up } D
$$$$
(n=6)
$$$$
\text { DDT }(35 \mathrm{mg} / \mathrm{kg})
$$$$
\text { DM }(0.5 \mathrm{mg} / \mathrm{kg})
$$$$
p-\mathrm{NP}(2.5 \mu \mathrm{g} / \mathrm{kg})
$$$$
\text { Genistein }(2.5 \mu \mathrm{g} / \mathrm{kg})
$$$$
\text { Zearalenone (2.5 }
$$$$
\mu \mathrm{g} / \mathrm{kg} \text { ) }
$$$$
\text { Coumestrol }(2.5 \mu \mathrm{g} / \mathrm{kg})
$$

Figure 1: Experimental design describing the life time (in utero-, lactational- and direct) exposure to either cottonseed oil, DDT, DDE or a mixture of EDCs. Exposure groups in both P1 and F1 generation, including the sample size, chemical doses, dosing duration and termination endpoints are indicated. 


\subsection{Chemicals and dosing procedure}

Four experimental groups were used in this study:

Group A - Control group - Cottonseed oil [Sigma-Aldrich, Steinheim, Germany; catalogue number: C7767, CAS Number: 8001-29-4] as vehicle;

Group B - DDT-exposed group - $35 \mathrm{mg} / \mathrm{kg}$ (DDT) [Sigma-Aldrich, Steinheim, Germany; catalogue number: 50-29-3];

Group C - DDE-exposed group - 35 mg/kg (DDE) [Sigma-Aldrich, Steinheim, Germany; catalogue number: 123897, CAS Number 72-55-9];

Group D - mixture-exposed group - 35 mg/kg DDT [Sigma-Aldrich, Steinheim, Germany; catalogue number: 50-29-3], 0.5 mg/kg Deltamethrin (DM) ([Chem Service, West Chester, PA, USA; catalogue number: PS-2071], $2.5 \mu \mathrm{g} / \mathrm{kg} p$-NP [Sigma-Aldrich, Steinheim, Germany; catalogue number: 290858, CAS Number 84852-15-3], $2.5 \mu \mathrm{g} / \mathrm{kg}$ coumestrol [Sigma-Aldrich, Steinheim, Germany; catalogue number: 27885, CAS Number 479-13-0], $2.5 \mu \mathrm{g} / \mathrm{kg}$ genistein [Sigma-Aldrich, Steinheim, Germany; catalogue number: G6776, CAS Number 446-72-0] and $2.5 \mu \mathrm{g} / \mathrm{kg}$ zearalenone [Sigma-Aldrich, Steinheim, Germany; catalogue number: Z2125, CAS Number 17924-92-4].

Doses were determined from previously published studies documenting exposure to chemicals present in malaria areas in South Africa $[6,8,9,11,21,22]$. Blood plasma levels expressed as lipid adjusted concentrations of $p, p^{\prime}$-DDT and $p, p^{\prime}$-DDE have been used as indicators of exposure [9,22]. High concentrations of $p, p^{\prime}$-DDT $(90.23 \pm 102.4 \mu \mathrm{g} / \mathrm{g})$ [9], 109.2 $\pm 106.6 \mu \mathrm{g} / \mathrm{g}$ [22] $)$ and $p, p^{\prime}-\operatorname{DDE}(215.47 \pm 210.6 \mu \mathrm{g} / \mathrm{g}$ [9], $\left.246.2 \pm 218.5 \mu \mathrm{g} / \mathrm{g} 22]\right)$ were found in the blood plasma of men living in the Limpopo Province of South Africa. High concentrations of $p, p^{\prime}-\mathrm{DDT}(45094.4 \pm 2579.5 \mu \mathrm{g} / \mathrm{kg})$ and $p, p^{\prime}-\mathrm{DDE}(192024.2 \pm 35892.3$ $\mu \mathrm{g} / \mathrm{kg}$ ) were measured in chicken fat samples [6]. DDT concentrations have been measured in the muscle $(700.0 \mu \mathrm{g} / \mathrm{kg})$, fat $(240,000.0 \mu \mathrm{g} / \mathrm{kg})$ and liver $(1600.0 \mu \mathrm{g} / \mathrm{kg})$ tissues of chickens [23]. Mean DDT concentrations of 18,11 , and $9.5 \mathrm{mg} / \mathrm{kg}$ in breast milk were reported for three DDT-sprayed villages in South Africa [24] including the highest DDT concentration level ever reported for breast milk from South Africa (140mg/kg) [23]. The 
dosages chosen in this study mimicked the high levels of DDT exposure measured in South Africa. All chemical substances were administered by oral gavage at a volume of $1 \mathrm{ml} / \mathrm{kg}$, which was calculated daily and adjusted for body mass.

F1 males were exposed in utero for 14 days, during lactation for 20 days (Postnatal day (PND) 1 - PND 20) and directly for 70 days (PND 21- PND 90). After dosing at PND 90, the adult F1 males were euthanized with an overdose of isoflurane by insufflation (Isofor $\AA$, Safeline Pharmaceutical [Pty] Ltd., South Africa) under controlled conditions.

\subsection{Tissue collection and histology preparation}

After termination, the anogenital distance (AGD) and body mass was measured. The organs were excised and weighed separately. The left and right testes were excised and the epididymis were separated from each testis and weighed individually and the relative testis weight was calculated. The relative testis weight represents the total testis weight relative to the total body weight, expressed as a percentage. The liver was weighed and the relative liver weight was calculated. The relative liver weight represents the total liver weight relative to the total body weight, expressed as a percentage.

The testes, epididymis, seminal vesicles and liver were fixed in Bouin's Fluid [15 parts Picric acid (BDH laboratory Supplies, Poole BH15 1TD, England); 5 parts 40\% Formalin (Merck, Darmstadt, Germany); 1 part Glacial acetic Acid (Merck, Darmstadt, Germany)], following standard protocols [24]. The tissues were embedded in paraffin blocks and sections of $4 \mu \mathrm{m}$ thick were made and collected on SuperFrost slides (Menzel-Glaser, Germany; catalogue number: J1800AMNZ). Slides were stained with Haematoxylin and Eosin ( $H$ \& E), for histological assessment. In each rat testis, 30 randomly selected seminiferous tubules were selected and the seminiferous tubule diameter, seminiferous epithelial thickness and lumen diameter were measured. Testicular tissue sections of the F1 male rats were viewed at $10 \mathrm{x}$ and $40 \mathrm{x}$ magnification, using a Nikon $\mathrm{BH}-2$ microscope fitted 
with a CC-2 digital camera, coupled to a computer with AnalySIS Imaging Processing software (Soft Imaging System, Münster, Germany).

\subsection{Epididymal sperm count}

The left cauda epididymis was used to determine the sperm concentration. The cauda epididymis was separated from the caput-corpus and placed in a petri dish containing $2 \mathrm{ml}$ phosphate buffered saline (PBS). The cauda epididymis was macerated to expel the sperm into the medium, which was then transferred to a Falcon tube. With the use of the Neubauer method, the sperm count was expressed as million/ml [25].

\subsection{Total testosterone radioimmunoassay procedure}

Blood was collected via cardiac puncture prior to death and collected in additive free tubes. The blood was centrifuged and the plasma stored at $-80^{\circ} \mathrm{C}$. The assay was performed according to the manufacturer's instructions (Immunotech, Marseille, France: Cat number IM1087). The limit of sensitivity for this total testosterone assay is $0.04 \mathrm{ng} / \mathrm{ml}$. The intraassay coefficient of variation is $8.6 \%$ and the inter-assay coefficient of variation is $11.9 \%$.

\subsection{Statistical analysis}

F1 males from the same litter share a common mother, a P1 female, and hence data analysis employed the Survey command in STATA 12 (StataCorp, TX, USA) [26] to deal with the dependence of data within litters (i.e. clusters). In total 16 clusters of F1 males were analyzed using Survey Linear Regression. The exposed groups (groups B-D) were compared to the control group (Group A), at the 0.05 level of significance. Additionally, group B was compared to group D to assess the possible effect of exposure to a single chemical compared to exposure of the same chemical in a mixture. Furthermore, differences among the exposed groups were assessed using the adjusted Wald Test at the 0.05 level of significance. Groups were compared with respect to endpoint values of the study 
parameters. In the analysis of the endpoint AGD, the value at baseline and the body weight was adjusted for.

\section{Results}

\subsection{Anogenital distance}

Shorter AGDs were recorded in the mixture-exposed group $(15.20 \mathrm{~mm} ; P=0.005)$ compared to the control group $(17.54 \mathrm{~mm})$. Although not statistically significant, the DDTexposed group $(18.55 \mathrm{~mm} ; P=0.863)$ had a longer mean AGD; whilst the DDE-exposed group (17.33mm; $P=0.360)$ had a marginally shorter mean AGD compared to the control (Table 1).

\subsection{Body mass}

The mean body mass $(\mathrm{g})$ of the F1 males is summarized in Table 1 . The survey linear regression indicated no difference between the body mass of control group compared to the DDT-exposed group (Group B: $P=0.561$ ), the DDE-exposed group (Group C: $P=$ 0.317) and the mixture-exposed group (Group D: $P=0.499)$.

\subsection{Liver}

The mean liver mass was higher in the DDT-exposed group $(P<0.001)$, the DDEexposed group $(P=0.003)$ and the mixture-exposed group $(P=0.03)$. Compared to the control group (17.36g), the DDT-exposed group (21.16g) had the largest mean liver mass, followed by the DDE group (20.65g) and then the mixture-exposed group (19.45g) (Table 1). Lipid droplet formation was observed in the liver tissue in the exposed groups. The DDEexposed group had the greatest presence of lipid droplets as well as abnormal cellular organization. The histology of the liver in the control group revealed no abnormal tissue morphology (Fig 2). 

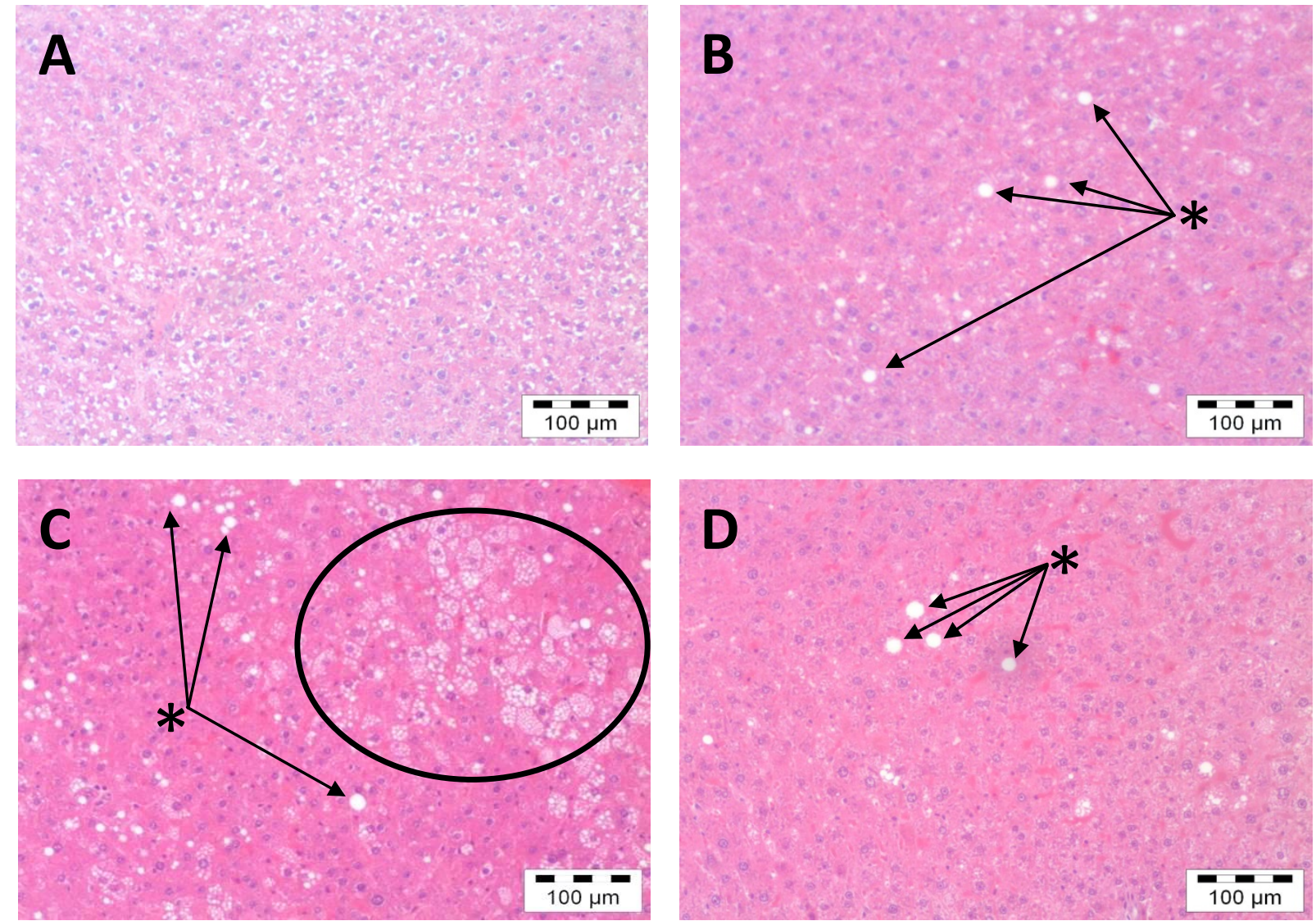

Figure 2: Histology of the liver showing lipid droplets in the liver of rats in the exposed groups at PND 90. A: Control group (cottonseed oil); B: DDT-exposed group lipid droplets $\left(^{*}\right)$; C: DDE-exposed group showing abnormal liver histology indicated by the black ring and lipid droplets $\left.{ }^{*}\right)$; D: mixture-exposed group with the presence of lipid droplets $\left(^{*}\right)$. 
Relative liver weight of control group (4.028) was less than the DDT-exposed group (4.962, $P<0.001)$, DDE-exposed group $(4.837 P<0.001)$ and mixture-exposed group (4.642, $P=0.001)$ was observed.

\subsection{Male accessory glands and epididymis}

A larger prostate mass was noted in the DDT-exposed group $(1.02 \mathrm{~g} ; P=0.018)$ compared to the control group $(0.83 \mathrm{~g})$. Although not significant, the DDE-exposed group (0.82g; $P=0.858)$ had a lower prostate mean mass compared to the control group (Table 1$)$. There was no difference between the mean seminal vesicle mass and the epididymal mass of the control group and the DDT-exposed group, the DDE-exposed group and the mixtureexposed group (Table 1).

\subsection{Testes}

The mean testes mass of the DDT-exposed group (3.88g; $P=0.019$ ), the DDEexposed group (3.95g; $P=0.047$ ), and the mixture-exposed group (4.02g; $P<0.001)$ was significantly larger than the control group (3.684g). No difference between the mean relative testis weight of the control group (0.86) compared to the DDT-exposed group $(0.89 ; P=$ $0.435)$ was observed. Relative testis weights in the DDE-exposed group $(0.96 ; P=0.036)$ and the mixture-exposed group $(0.97 ; P=0.016)$ were greater than the control group.

A histological examination of the testes showed selected seminiferous tubules containing dilated tubular lumens, marked detachment of the seminiferous tubule, necrosis in the interstitium, marked disorganization of the seminiferous epithelium with few germ cells present, reduced seminiferous tubule diameter with no lumen, absent seminiferous tubules and decreased cellularity of the seminiferous epithelium in the exposure groups (groups BD) (Fig 3). In the exposure groups, the changes in the size of the seminiferous tubule diameter, epithelium thickness and lumen diameter per stage of the spermatogenic cycle differed from the control (Figure 3). Although all stages were present, seminiferous tubules 


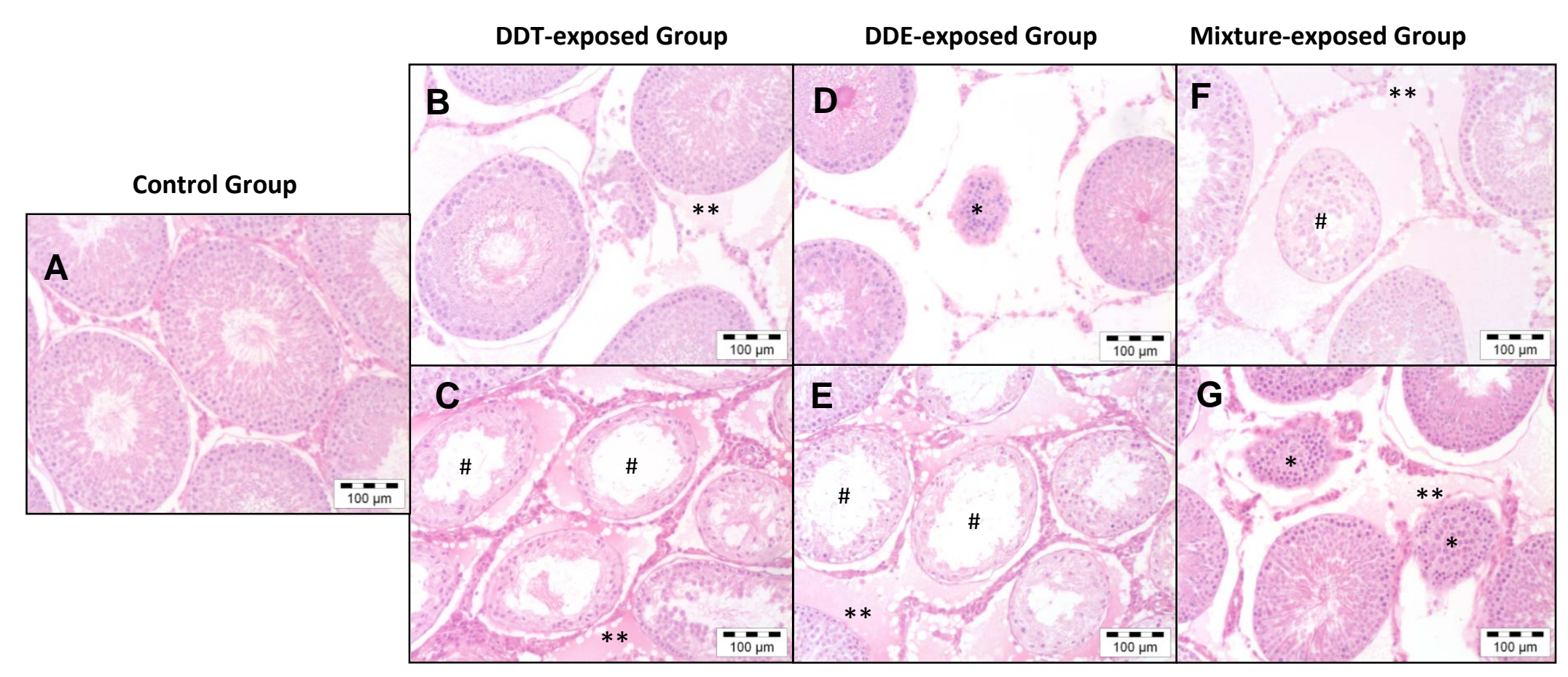

Figure 3: Testicular histology of F1 males at PND 90 - Normal testicular histology in the control group (A), abnormal testicular histology in DDT-exposed group (B-C), DDE-exposed group (D-E) and in the mixture-exposed group (F-G); small seminiferous tubule diameter with no lumen $\left({ }^{*}\right)$, necrosis in the interstitium $\left({ }^{*}\right)$, disorganization of the seminiferous epithelium $\left({ }^{*}\right)$. 
Table 1: The effect of various mixtures on reproductive parameters of the F1 males in the four experimental groups. Mean \pm SD

\begin{tabular}{|c|c|c|c|c|c|c|c|c|c|}
\hline & & Group A & Group B & $A: B$ & Group C & $A: C$ & Group D & A:D & B:D \\
\hline & Vallavie & $n=24^{a}$ & $\mathrm{n}=11^{\mathrm{b}}$ & $P$-value & $n=27^{c}$ & $P$-value & $n=15^{d}$ & $P$-value & $P$-value \\
\hline \multirow{2}{*}{ Measurements } & Anogenital distance $^{\#}(\mathrm{~mm})$ & $17.54 \pm 0.65$ & $18.55 \pm 0.17$ & 0.274 & $17.33 \pm 0.41$ & 0.707 & $15.20 \pm 0.16$ & $0.001^{*}$ & $0.005^{*}$ \\
\hline & Body mass (g) & $430.34 \pm 34.92$ & $437.54 \pm 23.94$ & 0.561 & $414.91 \pm 32.15$ & 0.317 & $419.08 \pm 32.74$ & 0.499 & 0.184 \\
\hline \multirow{7}{*}{ Mass } & Liver mass (g) & $17.36 \pm 2.16$ & $21.16 \pm 1.29$ & $<0.001^{*}$ & $20.65 \pm 5.06$ & $0.003^{*}$ & $19.45 \pm 2.00$ & $0.031^{*}$ & $0.025^{*}$ \\
\hline & Relative liver weight & $4.028 \pm 0.31$ & $4.837 \pm 0.19$ & $<0.001 *$ & $4.962 \pm 1.01$ & $<0.001^{*}$ & $4.642 \pm 0.33$ & $0.001^{*}$ & 0.145 \\
\hline & Prostate (g) & $0.83 \pm 0.24$ & $1.02 \pm 0.20$ & 0.018 * & $0.82 \pm 0.23$ & 0.858 & $0.83 \pm 0.21$ & 0.981 & 0.065 \\
\hline & Seminal vesicles mass $(g)$ & $1.46 \pm 0.37$ & $1.60 \pm 0.43$ & 0.294 & $1.57 \pm 0.47$ & 0.430 & $1.58 \pm 0.35$ & 0.494 & 0.886 \\
\hline & Epididymal mass (g) & $1.47 \pm 0.26$ & $1.59 \pm 0.25$ & 0.227 & $1.42 \pm 0.30$ & 0.530 & $1.44 \pm 0.19$ & 0.721 & 0.110 \\
\hline & Testicular mass (g) & $3.68 \pm 0.22$ & $3.88 \pm 0.16$ & $0.019^{*}$ & $3.95 \pm 0.32$ & $0.047^{*}$ & $4.02 \pm 0.31$ & $<0.001^{*}$ & 0.092 \\
\hline & Relative testis weight & $0.86 \pm 0.08$ & $0.89 \pm 0.06$ & 0.435 & $0.96 \pm 0.08$ & $0.036^{*}$ & $0.97 \pm 0.10$ & $0.016^{*}$ & 0.823 \\
\hline \multirow{3}{*}{ Histology } & Seminiferous tubule diameter $(\mu \mathrm{m})$ & $295.42 \pm 19.25$ & $260.65 \pm 17.98$ & $<0.001^{*}$ & $260.00 \pm 14.53$ & $<0.001^{*}$ & $257.78 \pm 9.36$ & $<0.001^{*}$ & $0.028 *$ \\
\hline & Seminiferous epithelium thickness $(\mu \mathrm{m})$ & $100.40 \pm 8.58$ & $84.77 \pm 3.45$ & $<0.001 *$ & $86.33 \pm 4.10$ & $<0.001 *$ & $82.40 \pm 8.45$ & $<0.001 *$ & 0.622 \\
\hline & Lumen diameter $(\mu \mathrm{m})$ & $106.84 \pm 20.38$ & $87.62 \pm 12.40$ & $<0.001^{*}$ & $80.15 \pm 8.08$ & $<0.001^{*}$ & $96.34 \pm 19.48$ & $<0.001^{*}$ & 0.852 \\
\hline Sperm count & Total sperm count $\left(\times 10^{6} / \mathrm{ml}\right)$ & $48.46 \pm 14.36$ & $60.13 \pm 17.50$ & 0.063 & $50.69 \pm 16.47$ & 0.685 & $38.72 \pm 12.34$ & 0.090 & $0.010^{*}$ \\
\hline Hormone & Testosterone (nmol/L) & $21.33 \pm 1.74$ & $23.06 \pm 3.01$ & 0.392 & $28.12 \pm 3.53$ & $0.038^{*}$ & $28.62 \pm 2.96$ & $0.023^{*}$ & 0.203 \\
\hline
\end{tabular}

Group A = Cottonseed oil; Group B = $35 \mathrm{mg} / \mathrm{kg} \mathrm{DDT;} \mathrm{Group} \mathrm{C} \mathrm{=} 35 \mathrm{mg} / \mathrm{kg}$ DDE; Group D = $35 \mathrm{mg} / \mathrm{kg}$ DDT + 0.5mg/kg DM + 2.5 $\mu \mathrm{g} / \mathrm{kg} 4-\mathrm{NP}+2.5 \mu \mathrm{g} / \mathrm{kg}$ Coumestrol, $2.5 \mu \mathrm{g} / \mathrm{kg}$

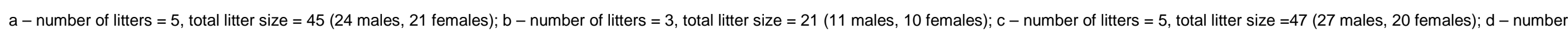
of litters $=4, d-$ number of litters $=4$, total litter size $=32$ ( 15 males, 17 females)

$*=0.05$

\# corrected for body weight 
had thinner epithelium thickness suggesting reduced germ cell layers. This is indicative of Sertoli cell toxicity resulting in altered fluid retention.

There was no difference between the total cauda epididymal sperm count of the control group compared to the DDT-exposed group $(P=0.063)$, the DDE-exposed group ( $P$ $=0.685)$, and the mixture-exposed group $(P=0.090)$ (Table 1$)$. Although not statistically significant, the mixture-exposed group $\left(38.72 \times 10^{6}\right)$ had the lowest total cauda epididymal sperm count.

There were higher testosterone concentrations in the DDE-exposed group $(28.12 \mathrm{nmol} / \mathrm{L} ; P=0.038)$ and the mixture-exposed group $(28.612 \mathrm{nmol} / \mathrm{L} ; P=0.023)$. However, there was no statistically significant difference between the testosterone concentration of the control group $(21.33 \mathrm{nmol} / \mathrm{L})$ compared to the DDT-exposed group (23.06nmol/L; $P=0.392)$.

\section{Discussion}

This study investigated the effects of life time exposure (in utero-, lactational- and direct) to environmentally relevant concentrations of EDCs present in a South African malaria area, using the rat model. The chemicals and doses were representative of a possible real-life exposure scenarios that males living in a malaria area may encounter throughout their development. Exposure to EDCs associated with common pesticides and agricultural chemicals resulted in significantly shorter AGDs in the mixture-exposed rats (group 4), significantly higher liver mass in the DDT-, DDE- and mixture-exposed rats (groups 2-4, respectively) and the presence of lipid droplets in the hepatic tissue. A significantly higher testicular mass was observed in the DDT-, DDE- and mixture-exposed rats, with testicular histology showing apical sloughing, reduced seminiferous tubule diameters and disorganization of the seminiferous epithelium. Additionally, a significantly higher total testosterone concentration was found in the DDE- and mixture-exposed rats. 
In animals [27] and humans [28] the AGD is used as a marker for genital development [29]. The male AGD is generally twice as long as female AGD in several mammalian species [30]. The narrow masculinization programming window during prenatal development is sensitive to androgen action [31]. A shorter AGD in males indicates feminization and a disturbance in the androgen to estrogen ratio in the uterus [32]. The shorter AGD at PND 90 (this study) was observed in the mixture-exposed rats (group D) which received technical grade DDT, DM, $p$-NP and phytoestrogens, all of which have estrogenic properties. The shorter AGD may be ascribed to lower androgen function during the hormone-sensitive male programming window [33] or postnatally [34]. Thus, the mixture of the substances used in this study may have had an additive or synergistic effect resulting in a shorter AGD in the mixture-exposed group (group D). A similar effect was observed in rats exposed to mixtures of pesticides commonly used in Denmark and Europe [35]. The testicular dysgenesis syndrome (TDS) includes cryptorchidism, hypospadias, poor semen quality and testicular germ cell tumors [36]. Since AGD is a biomarker of androgen action in fetal life that continues into adult life, it is now considered to be a part of the TDS [28] and may form part of routine human male reproductive health endpoints in future studies.

Enlargement of the liver is often reported in toxicology studies and is a useful marker of the effects of pesticides and their metabolites [37]. In this study, the mean liver mass in the exposed rats (groups B-D) was significantly higher than the rats in the control group (Table 1), similar to reports in literature $[38,39]$. The higher liver mass after exposure to environmentally relevant doses is concerning as enlargement of the liver is associated with hyperplasia ultimately leading to hepatocellular hypertrophy. Hepatocyte hypertrophy following chemical exposure is the most common cause of increases in absolute and relative liver mass [40]. Mild steatoses was observed in the liver tissue of the DDT-, DDE- and mixture-exposed rats (groups B-D, respectively)(Fig 2). The mild steatosis may be ascribed to chemical interference with lipid mobilization during the formation of very low density lipids (VLDL) [41]. p,p'-DDE has been detected in VLDL of males occupationally exposed to DDT 
[42] suggesting that lipoprotein production is an EDC target. Thus life time exposure (in utero-, lactational- and direct) to EDCs may increase lipid synthesis in the hepatocytes and lead to steatosis, negatively affecting the functioning of the liver and general health.

Exposure to EDCs, particularly in utero exposure to DDT, results in altered fertility in adult rats due to increases in prostate mass and reductions in accessory organ mass [43]. The higher prostate mass measured in the DDT-exposed rats (group B) (Table 1) can be linked to permanent disruptions in prostate growth associated with elevated endogenous or exogenous estrogenic compounds [44]. Since technical grade DDT has estrogenic properties mainly due to the $o, p^{\prime}-\mathrm{DDT}$ isomer [45], the increase in prostatic growth in the DDT-exposed rats might be mediated through estrogen receptors and this needs further investigation. Rats exposed to DDE had smaller prostate glands than rats in the control group. This result was not significant, but interesting since the anti-androgenic effect of DDE in lowering the prostate mass has been reported before [46].

The greater mean testicular mass in the DDT-exposed rats (group B); the DDEexposed rats (group C) and in the mixture-exposed rats (group D) (Table 1) can be attributed to the dilation of the tubular lumen [48]. The Sertoli cell produces the seminiferous tubule fluid which is absorbed by the rete testis, efferent ducts and the epididymal epithelium [47]. The tubular fluid volume is a function of the secretion and reabsorption rates in the rete testis and epididymis. Changes in these functions may manifest as dilated tubular lumens [47] increasing the testis mass. Both increases and decreases in testicular mass have been reported following exposure to various EDCs at various exposure durations and doses [48, 49].

The pathogenesis of toxicant induced testicular injury, possibly leading to Sertoli cell toxicity can be investigated using histological methods [50]. In this study, all rats exposed to individual pesticides and mixtures of pesticides had dilated tubular lumens, marked detachment of the seminiferous tubule, necrosis in the interstitium, marked disorganization 
of the seminiferous epithelium with few germ cells present, reduced seminiferous tubule diameter with no lumen, absent seminiferous tubules and decreased cellularity of the seminiferous epithelium (Fig 3). The seminiferous tubule diameters and seminiferous epithelium thickness of the exposed rats were also smaller particularly in the DDE-exposed (group C) and mixture-exposed rats (group D). Even though the seminiferous tubule diameter and the epithelium thickness of exposed rats was smaller, relatively larger luminal sizes were measured. Testicular histology studies investigating the effects of EDC exposures $[8,48]$ have associated larger luminal sizes with Sertoli cell toxicity which could negatively affect spermatogenesis. Lifespan exposure to EDCs at environmentally relevant concentrations used in this study may have the same negative effects on male fertility.

Despite the observed histological abnormalities, sperm counts of the exposed and control groups did not differ statistically (Table 1). Exposure to DDT [9, 51], DDE [51], DM [8] and $p$-NP [48] should result in decreased sperm counts. While epididymal sperm counts are commonly used in reproductive toxicology [52], only the number of sperm are taken into account and not the motility, morphology or any other exposure-induced defects. Although no significant change was observed in the sperm count, possible decreased motility and effects on sperm morphology cannot be ruled out.

Spermatogenesis is a hormonally dependent process that requires testosterone [53]. Rats exposed to DDE- (group C) and mixture-exposed rats (group D) (Table 1) had higher testosterone concentrations than control rats (group A) and DDT exposed rats (group B). Higher serum testosterone concentrations have been recorded in male rats exposed to $p, p^{\prime}$ DDE for 15 days [54]. Increases in steroid hormone binding globulin have been associated with estrogenic and anti-androgenic compounds and DDT uptake in men [54]. The increase in testosterone and SHBG could result from a 'functional' androgen deficiency [55]. Possible stimulation of the $\mathrm{GnRH}$ in the hypothalamus through a positive feedback loop could increase testosterone levels in individuals with suppressed androgen activity. 
The "something from nothing" principle proposes that exposure to a single chemical may have no observed effects. However, when there is exposure to several of these chemicals in a mixture, significant effects may occur [18]. These mixtures may even have significant effects at lower concentrations than the "no observed adverse effect levels" (NOAELS) reported for individual chemicals [35]. Although rats in the control group had significantly different endocrine sensitive endpoints to all exposure groups (Table 1), differences were also observed within the exposure groups. Rats exposed to the chemical mixture had shorter anogenital distances, heavier livers, less sperm and smaller seminiferous tubules when compared to rats exposed to individual chemicals (Table 1). The interaction of chemicals in mixtures may act through different mechanisms [56] suggesting the importance of investigating effects of chemical mixtures. Exposure to environmentally relevant concentrations of chemical mixtures found in a malaria area need to be explored. The complex agro-economic environment encountered in the Vhembe district of Limpopo is conducive to intensive use of agricultural chemicals and extensive malaria vector control [6, $21,57]$.

The data from this study indicate that lifetime (in utero-, lactational- and direct) exposure to DDT, DDE, DM, $p$-NP and phytoestrogens have a negative influence on male reproductive health and associated endocrine-sensitive endpoints. Since these EDCs, in particular DDT and DDE have long half-lives, the long-term and thus multiple-generational effects need to be addressed in future studies. From these results, the testes are the clear targets of the selected EDCs used in this study. The abnormal testicular histology with apical sloughing and seminiferous tubule disorganization warrants investigation into the possible molecular and biochemical mechanisms and changes that a lifetime of exposure to these EDCs may have on testicular function. In particular, the results from this study suggest further investigation into the effect that exposure to the selected EDCs may have on the Sertoli cells. Since this reproductive toxicology study constitutes lifetime (in utero-, lactational 
and direct) exposure to environmentally relevant concentrations of EDCs present in a malaria area, these results might represent human exposures.

\section{Conflict of interest}

The authors declare that there are no conflicts of interest.

\section{Acknowledgments}

The Medical Research Council of South Africa funded this study. We thank Prof PJ Becker of the Research Support Office in the Faculty of Health Sciences, University of Pretoria, for the statistical support.

\section{References}

[1] Bergman A, Heindel JJ, Jobling S, Kidd KA, Zoeller RT. State of the science of endocrine disrupting chemicals 2012. Geneva, Switzerland: United Nations Environment Programme and the World Health Organization; 2013.

[2] Frye CA. Endocrine-disrupting chemicals: elucidating our understanding of their role in sex and gender-relevant end points. Vitam Horm 2014;94:41-98.

[3] Fudvoye J, Bourguignon JP, Parent AS. Endocrine-disrupting chemicals and human growth and maturation: a focus on early critical windows of exposure. Vitam Horm 2014;94:1-25.

[4] Svechnikov K, Stukenborg JB, Savchuck I, Soder O. Similar causes of various reproductive disorders in early life. Asian J Androl 2014;16(1):50-59.

[5] De Falco M, Forte M, Laforgia V. Estrogenic and anti-androgenic endocrine disrupting chemicals and their impact on the male reproductive system. Front Environ Sci 2015;3(3).

[6] Bornman MS, Barnhoorn IEJ, Genthe B, van Vuuren JH, Pieterse GM, Aneck-Hahn $\mathrm{NH}$, et al. DDT for malaria control: effects in indicators and health risk. Pretoria: University of Pretoria, 2010 February 2010. Report No.: Contract No.: 1674/1/09.

[7] Eskenazi B, Chevrier J, Rosas LG, Anderson HA, Bornman MS, Bouwman H, et al. The Pine River statement: human health consequences of DDT use. Environ Health Perspect 2009;117(9):1359-1367.

[8] Kilian E, Delport R, Bornman MS, de Jager C. Simultaneous exposure to low concentrations of dichlorodiphenyltrichloroethane, deltamethrin, nonylphenol and phytoestrogens has negative effects on the reproductive parameters in male SpraqueDawley rats. Andrologia 2007;39(4):128-135. 
[9] Aneck-Hahn NH, Schulenberg GW, Bornman MS, Farias P, Reif S, de Jager C. Impaired semen quality associated with environmental DDT exposure in young men living in a malaria area in the Limpopo Province, South Africa. J Androl 2007;28:423-434.

[10] de Jager C, Myburgh J, van der Burg B, Lemmen JG, Bornman MS, editors. Estrogenic contamination of South African river waters: a pilot study. Endocrine Disruptor and the Water Industry Symposium; 2002 18-20 April; Cincinnati, Ohio, USA.

[11] Dalvie MA, Myers JE, Lou Thompson M, Dyer S, Robins TG, Omar S, et al. The hormonal effects of long-term DDT exposure on malaria vector-control workers in Limpopo Province, South Africa. Environ Res 2004;96(1):9-19.

[12] World Health Organization. World Malaria Report 2011. Geneva: World Health Organization, 2011.

[13] Yoon K, Kwack SJ, Kim HS, Lee BM. Estrogenic endocrine-disrupting chemicals: molecular mechanisms of actions on putative human diseases. J Toxicol Environ Health $B$ Crit Rev 2014;17(3):127-174.

[14] World Health Organization. World Malaria Report 2013. Geneva: World Health Organization, 2013.

[15] Office of Environmental Health Hazard Assessment. Toxicological profile for nonylphenol. CA, USA: 2009 07-055.

[16] de Jager $C$. The possible effects of p-nonylphenol, an environmental toxicant with estrogenic properties, on fertility [PhD Thesis]. Pretoria: University of Pretoria; 1996.

[17] Stoev SD. Foodborne mycotoxicoses, risk assessment and underestimated hazard of masked mycotoxins and joint mycotoxin effects or interaction. Environ Toxicol Pharmacol 2015;39(2):794-809.

[18] Silva E, Rajapakse N, Kortenkamp A. Something from "nothing" - Eight weak estrogenic chemicals combined at concentrations below NOECs produce signifcant mixture effects. Environ Sci Technol 2002;36:1751-1756.

[19] SABS. The Care and Use of Animals for Scientific Purposes: SANS 10386. Pretoria, South Africa: South African Bureau of Standards, Standards Division., 2008.

[20] OECD. OECD Guidelines for the testing of chemicals. One-generation reproductive toxicity study (Protocol 415). Organization for Economic Co-operation and Development,, 19832 May 1983. Report No.: 415.

[21] Van Dyk JC, Bouwman H, Barnhoorn IE, Bornman MS. DDT contamination from indoor residual spraying for malaria control. Sci Total Environ 2010;408(13):2745-2752.

[22] de Jager $\mathrm{C}$, Aneck-Hahn NH, Bornman MS, Farias $\mathrm{P}$, Leter $\mathrm{G}$, Eleuteri $\mathrm{P}$, et al. Sperm chromatin integrity in DDT-exposed young men living in a malaria area in the Limpopo Province, South Africa. Hum Reprod 2009;24(10):2429-2438.

[23] Bouwman H, Kylin H, Sereda B, Bornman R. High levels of DDT in breast milk: Intake, risk, lactation duration, and involvement of gender. Environmental Pollution 2012;170:63-70. 
[24] Bancroft JD, Cook HC. Manual for histological techniques. Livingstone, New York: Churchill; 1984.

[25] World Health Organization. WHO laboratory manual for the examination of human semen and sperm-cervical musuc interaction. 4th ed. Cambridge, UK: Cambridge University Press; 1999.

[26] StataCorp. Stata Statistical Software: Release 12. 12 ed. College Station, TX: StataCorp LP; 2011.

[27] Gray LE, Jr., Ostby JS, Kelce WR. Developmental effects of an environmental antiandrogen: the fungicide vinclozolin alters sex differentiation of the male rat. Toxicol Appl Pharmacol 1994;129:46-52.

[28] Swan SH, Main KM, Liu F, Stewart SL, Kruse RL, Calafat AM, et al. Decrease in Anogenital Distance among Male Infants with Prenatal Phthalate Exposure. Environmental Health Perspectives 2005;113(8):1056-1061.

[29] Eisenberg ML, Hsieh MH, Walters RC, Krasnow R, Lipshultz LI. The relationship between anogenital distance, fatherhood, and fertility in adult men. PLoS One 2011;6(5):e18973.

[30] Hsieh MH, Breyer BN, Eisenberg ML, Baskin LS. Associations among hypospadias, cryptorchidism, anogenital distance, and endocrine disruption. Curr Urol Rep 2008;9(2):137142.

[31] Sharpe R. Male reproductive health disorders and the potential role of exposure to environmental chemicals. United Kingdom: CHEMTrust, 2009.

[32] Mitchell RT, Mungall W, McKinnell C, Sharpe RM, Cruickshanks L, Milne L, et al. Anogenital Distance Plasticity in Adulthood: Implications for Its Use as a Biomarker of Fetal Androgen Action. Endocrinology 2015;156(1):24-31.

[33] Welsh M, Saunders PTK, Fisken M, Scott HM, Hutchison GR, Smith LB, et al. Identification in rats of a programming window for reproductive tract masculization, disruption of which leads to hypospadias and cryptorchidism. J Clin Investigation 2008;118:1479-1490.

[34] Cederroth CR, Zimmermann C, Nef S. Soy, phytoestrogens and their impact on reproductive health. Mol Cell Endocrinol 2012;355(2):192-200.

[35] Hass U, Boberg J, Christiansen S, Jacobsen PR, Vinggaard AM, Taxvig C, et al. Adverse effects on sexual development in rat offspring after low dose exposure to a mixture of endocrine disrupting pesticides. Reproductive Toxicology 2012;34(2):261-274.

[36] Skakkebaek NE, Rajpert-De Meyts E, Main KM. Testicular dysgenesis syndrome: an increasingly common developmental disorder with environmental aspects. Hum Reprod 2001;16(5):972-978.

[37] Andrew D. Pesticides Safety Directorate (PSD) Guidance Document: Interpretation of liver enlargement in regulatory toxicity studies. York, England, . , 2005.

[38] Kirman CR, Aylward LL, Hays SM, Krishnan K, Nong A. Biomonitoring equivalents for DDT/DDE. Regul Toxicol Pharmacol 2011;60(2):172-180. 
[39] ASTDR. Toxicological profile for DDT, DDE and DDD. Atlanta, GA, USA: Agency for Toxic Substances and Disease Registry, US Department of Health and Human Services, 2002.

[40] Maronpot RR, Yoshizawa K, Nyska A, Harada T, Flake G, Mueller G, et al. Hepatic enzyme induction: histopathology. Toxicol Pathol 2010;38(5):776-795.

[41] Spiller GA. Handbook of Lipids in Human Nutrition. Boca Raton, FL, USA: CRC Press; 1995. 256 p.

[42] Ljunggren SA, Helmfrid I, Salihovic S, van Bavel B, Wingren G, Lindahl M, et al. Persistent organic pollutants distribution in lipoprotein fractions in relation to cardiovascular disease and cancer. Environ Int 2014;65:93-99.

[43] Damstra T, Barlow S, Bergman A, Kavlock R, van der Kraak G. Global assessment of the state-of-the-science of endocrine disruptors. Geneva, Switzerland: International Programme on Chemical Saftey: World Health Organization, 2004.

[44] Huang L, Pu Y, Alam S, Birch L, Prins GS. Estrogenic regulation of signaling pathways and homeobox genes during rat prostate development. J Androl 2004;25(3):330337.

[45] Metcalf RL. Insect control technology. In: Kroschwitz J, Howe-Grant M, editors. KirkOthmer encyclopedia of chemical technology. New York: John Wiley and Sons, Inc; 1995. p. 524-602.

[46] Prins GS. Endocrine disruptors and prostate cancer risk. Endocr Relat Cancer 2008;15(3):649-656.

[47] Creasy DM. Pathogenesis of male reproductive toxicity. Toxicol Pathol 2001;29(1):64-76.

[48] de Jager C, Bornman MS, Oosthuizen JMC. The effect of p-nonylphenol on the fertility potential of male rats after gestational, lactational and direct exposure. Andrologia 1999;31:107-113.

[49] Jacobsen PR, Axelstad M, Boberg J, Isling LK, Christiansen S, Mandrup KR, et al. Persistent developmental toxicity in rat offspring after low dose exposure to a mixture of endocrine disrupting pesticides. Reproductive Toxicology 2012;34(2):237-250.

[50] Russell L, Griswold M. The sertoli cell. 1st ed. Clearwater, Florida, USA: Cache River Press; 1993. $801 \mathrm{p}$.

[51] de Jager C, Farias P, Barazza-Villarreal A, Avilla MH, Ayotte P, Dewailly E, et al. Breakthroughs in andrology: reduced seminal parameters associated with environmental DDT exposure and p,p'-DDE concentrations in men in Chiapas, Mexico: a cross-sectional study. J Androl 2006;27(1):16-27.

[52] Wang Y. Epididymal sperm count. In: Coruzzi G, editor. Current protocols in toxicology. Hoboken, New Jersey, USA: John Wiley \& Sons, Inc.; 2003. p. 16.16.1116.16.15.

[53] Nieschlag E, Behre H. Andrology: Male reporductive health and dysfunction. 2nd ed. Münster, Germany: Springer-Verlag; 2001. 
[54] O'Connor JC, Frame SR, Davis LG, Cook JC. Detection of the environmental antiandrogen $p, p^{\prime}$-DDE in CD and Long-Evans rats using a Tier 1 screening battery and a Hershberger Assay. Toxicol Sci 1999;51:44-53.

[55] Bornman MS, Delport R, Farias $\mathrm{P}$, Aneck-Hahn NH, de Jager C. Hormonal changes associated with DDT uptake in young males. International Society for Environmental Epidemiology; 13-16 September; Barcelona2011.

[56] ASTDR. Guidance manual for the assessment of joint toxic action of chemical mixtures (final). Atlanta, GA: Agency for Toxic Substances and Disease Registry, US Department of Health and Human Services, 2004.

[57] Bouwman H, Bornman R, van Dyk C, Barnhoorn I. First report of the concentrations and implications of DDT residues in chicken eggs from a malaria-controlled area. Chemosphere 2015;137:174-177. 